\title{
ACOUSTICS2008/1740 \\ An experimental method of corrugated surface reconstruction investigated in combination with finite-element simulation
}

\author{
S. W Herbison, E. Lamkanfi and N. F Declercq \\ Georgia Tech Lorraine - G.W. Woodruff School of ME, UMI Georgia Tech - CNRS 2958, 2 rue Marconi, \\ 57070 Metz, France
}

Current theory applied to ultrasonic diffraction from periodically corrugated surfaces is valid only in the far-field; it cannot be applied to determine the diffracted field within the surface corrugations themselves. Therefore, information regarding the corrugation is difficult to extract from experiments that are necessarily conducted in the far-field. The present work aims to simulate a potential experimental method for investigating the corrugations themselves. Using near-field results obtained from a finite-element code, the far-field diffraction is constructed as a series of time-harmonic plane waves. By back-propagating this diffracted field, information about the corrugations is obtained. Comparing the results of the back-propagated field to the field generated by the finite-element code shows the limitations of determining details of periodically corrugated surfaces from far-field experiments. 